Relations industrielles

Industrial Relations

\title{
Ressources humaines pour le développement industriel, B.I.T., Genève, 1967, 276 pp.
}

\section{Pierre Dionne}

Volume 23, numéro 1, 1968

URI : https://id.erudit.org/iderudit/027883ar

DOI : https://doi.org/10.7202/027883ar

Aller au sommaire du numéro

Éditeur(s)

Département des relations industrielles de l'Université Laval

ISSN

0034-379X (imprimé)

1703-8138 (numérique)

Découvrir la revue

Citer ce compte rendu

Dionne, P. (1968). Compte rendu de [Ressources humaines pour le développement industriel, B.I.T., Genève, 1967, 276 pp.] Relations industrielles / Industrial Relations, 23(1), 183-184. https://doi.org/10.7202/027883ar

Tous droits réservés @ C Département des relations industrielles de l'Université Laval, 1968
Ce document est protégé par la loi sur le droit d'auteur. L’utilisation des services d'Érudit (y compris la reproduction) est assujettie à sa politique d'utilisation que vous pouvez consulter en ligne.

https://apropos.erudit.org/fr/usagers/politique-dutilisation/ 
déjò le Manuel pratique de l'agent de maîtrise » publié en 1953 par Entreprise Moderne d'Edition, réédité à huit reprises et diffusé à plus de 80,000 exemplaires. Le présent ouvrage remplace ce dernier et est conçu dans le même esprit: un instrument de formation professionnelle pour les contremaitres.

Déjà vieux de quatorze ans, l'ancien manuel devait être modernisé et mis à jour. Depuis 1953, en effet, des changements sont survenus un peu partout: de nouveaux types de relations humaines ont été créés dans le milieu social et le milieu du travail, de nouvelles méthodes d'organisations sont apparues, de nouvelles techniques voient le jour.

Partant, les auteurs se rendent compte de la nécessité pour les agents de maîtrise de connaître ces changements tant pour leur bien-être que pour celui des autres. Les ouvriers qui entrent à l'usine sont de plus en plus qualifiés et responsables; l'agent de maitrise doit donc renouveler ses connaissances et même les agrandir de façon à ne pas perdre de vue ces travailleurs.

S'il ne veut pas se retrouver moins compétent que ceux qu'il dirige, l'agent de maîtrise ne peut pas ce complaire dans ses connaissances actuelles. Regarder les gens ovec des yeux neufs, satisfaire sa curiosité intellectuelle, savoir poser des questions, avoir l'esprit éveillé, voilà un homme qui évolue ovec son temps. Tous ces besoins que peut avoir un agent de maîtrise seront en grande partie satisfaits à la lecture de ce volume qui traite de trois sujets principaux: le métier de chef, l'organisation de l'entreprise, le rôle de l'agent de maîtrise.

La première partie de l'ouvrage "Le métier de chef définit ce qu'est une entreprise, situe le commandement et donne finalement les diverses fonctions du chef selon Foyol.

Lo deuxième partie traite de l'organisation générale de l'entreprise. On y retrouve les diverses fonctions de l'entreprise décrites très brièvement exception faite de la fonction technique étudiée sous tous ses angles: la conception du produit et sa réalisation, l'aménogement et l'entretien des biens de lo société, le prix de revient.

La troisième partie analyse le rôle de l'agent de maîtrise ; il ne serait pas nécessaire de spécifier que c'est la partie la plus importante de l'ouvrage. Tous les aspects du travail de l'agent de maîtrise sont étudiés. De la nécessité de la qualité du produit au besoin, pour l'agent de maîtrise, de connaî- tre ses hommes en passant par l'outillage utilisé, la formation du personnel, la sécurité au travail, chaque fois, les auteurs lui donnent des conseils utiles et des moyens efficaces pour qu'il puisse remplir le mieux possible son rôle d'agent de maîtrise.

En plus du précieux contenu de ce manuel, soulignons la présentation attrayante de ce livre: nombreuses illustrations, impression deux couleurs, reliure à anneaux.

\section{Rosaire ROY}

\section{Ressources humaines pour le développement industriel, B.I.T., Genève, 1967, 276 pp.}

Le présent volume rassemble une série de documents qui visent à cerner quelquesuns des principoux problèmes que les pays en voie de développement rencontrent au cours de leur industrialisation dans le domaine de la main-d'oeurre et des questions sociales, ainsi qu'à examiner les mesures à prendre, aux échelons national et international, pour résoudre ces problèmes.

Les documents contenus dans ce volume sont répartis en deux groupes. La première partie, intitulée "Le problème de la maind'oeuvre ", comprend quatre études consacrées aux problèmes de la formation professionnelle et de l'utilisation de la maind'oeuvre en vue du développement industriel. Les sujets suivants, entre autres, y sont examinés:

a) détermination des buts, quantitatifs et qualitatifs, à atteindre en matière d'enseignement et de formation professionnelle de la main-d'oeuvre industrielle qualifiée ;

b) établissement de systèmes cohérents et efficaces fondés sur une base législative et administrative adéquate et création d'organisations nationales de formation industrielle, en vue d'atteindre les objectifs ainsi définis;

c) rôle particulier de l'industrie elle-même dans le perfectionnement des qualifications professionnelles;

d) mesures à prendre pour utiliser au mieux lo main-d'oeuvre qualifiée disponible ;

e) possibilités d'action sur le plan international pour permettre aux pays en voie de développement d'élaborer et de mener à bien des programmes concrets de formation professionnelle en vue de l'industrialisation.

La deuxième partie du volume, intitulée * Autres questions exigeant une politique 
générale », contient également quatre études. Celle qui porte sur la participation sociale au développement industriel met l'accent sur l'importance d'une collaboration entre les différents groupes sociaux, tout porticulièrement les employeurs, les travailleurs et leurs organisations, et de leur porticipation active ou processus d'industrialisotion, puis passe en revue les fins auxquelles cette participation serait utile et les formes qu'elle peut revêtir. Celle qui est consacrée ¿े «l'emploi et l'industrialisation » expose un certoin nombre de raisons pour lesquelles, à moins de mesures adéquates, le secteur industriel des poys en voie de développement emploie parfois moins de main-d'oeuvre que ne le justifierait la situation économique et sociale de ces régions. Elle examine ensuite diverses possibilités de créer dans l'industrie un plus grand nombre d'emplois opprofondie dans le document suivant, intitulé « Recherche d'une technologie progressive pour les pays en voie de développement s. Enfin, le document portant sur les salaires et le développement industriel relève que, lorsqu'un pays s'efforce de parvenir à l'industrialisation, le succès de celle-ci et lc manière dont les avantages du développement industriel seront réportis dépendront en partie du niveau des solaires, de leur structure et de leur taux d'accroissement. La même étude montre quelles difficultés se présentent dans ce domaine.

II est évident que c'est surtout oux pays intéressés qu'il oppartient de faire le nécessaire pour résoudre les problèmes posés par I'industrialisation dans le domaine social et en matière de main-d'oeuvre. Cependant, certaines formes d'action internationale peuvent contribuer à surmonter les difficultés. On a donc jugé approprié, dans des documents préparés d̀ l'intention d'un colloque international, $d^{\prime}$ accorder une attention porticulière aux possibilités d'exercer une action utile sur le plan international. Trois genres de mesures prises à cet échelon peuvent renforcer les politiques nationales en matière de main-d'oeuvre industrielle: a) fourniture de services consultatifs, de matériel et de personnel technique qualifié, dons le cadre de programmes de coopération technique, bilatéraux et multilatéroux; b) assistance financière; c) action commune des pays en voie de développement qui cnt des problèmes analogues. Les documents rassemblés ci-après contiennent diverses suggestions portant sur des mesures de ces trois catégories. En ce qui concerne plus particulièrement la première de ces possibilités d'action internationale, on $y$ trouvera quelques renseignements sur les moyens dont dispose I'O.I.T. et la contribution qu'elle peut appor- ter aux Etats Membres, sur leur demande, pour étudier les aspects des plans d'industrialisation qui touchent aux questions sociales et d̀ la main-d'oeuvre.

\section{Pierre DIONNE}

\section{Technical Change and Manpower Planning,} by Salomon Barkin, O.C.D.E., Paris, 1967, 287 pp.

Cette brochure, la $4 \mathrm{e}$ d'une série intitulée \& Industrial Relations Aspect of Manpower Policy », cherche d̀ définir les principes qui ont guidé les entreprises privées dans leurs politiques de main-d'beurre face ou changement technologique.

Le volume compte 29 études de cas faites dans huit pays différents. Le choix des cas a été fait par une équipe d'experts en vue d'offrir une gamme très variée de types de changements ò l'intérieur de différentes industries.

Chaque étude apporte un nouvel élément de réponse ò des questions telles que:

-Quel degré de coordination existe-t-il entre les prévisions de changements technologiques et les efforts fournis en vue d'ajustements de la main-d'oeuvre?

-Quelles méthodes a-t-on employées pour faire les prédictions en maind'oeuvre et comment a-t-orı ajusté ces besoins à la force existante?

-A quel moment a-t-on eu recours à la consultation? $Y$ a-t-il eu riégociation?

-Quels ont été les avantages tirés du changement technique? Pour l'employeur? Pour l'employé?

Plus qu'une simple description, on cherche à montrer comment on a pu ou dans quelques cas comment on pourrait intégrer ces mesures individuelles à une politique de maind'oeuvre plus globale voir nationale. Cependant chaque cas demeure spécifique et ce n'est pas parce qu'une expérience a été profitable à une entreprise qu'on peut l'adopter comme une règle. II reste que ces études peuvent apporter un supplément aux politiques gouvernementales. La brochure prouve qu'une co-ordination des programmes est possible en vue d'établir une véritable politique de main-d'oeuvre où chacun (patronat, corps public, syndicat) a une meilleure compréhension de son rôle et de ses responsabilités.

Réjeonne MONETTE 